\title{
THEORETICAL NUCLEAR REACTION AND STRUCTURE STUDIES USING HYPERONS AND PHOTONS
}

Final Report

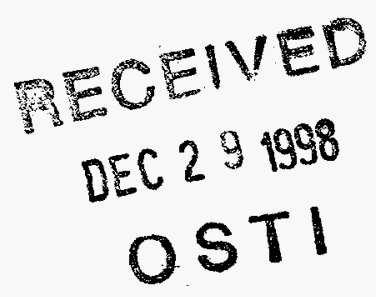

\author{
Stephen R. Cotanch \\ Department of Physics \\ North Carolina State University \\ Raleigh, North Carolina 27695-8202
}

PREPARED FOR THE U. S. DEPARTMENT OF ENERGY UNDER GRANT NUMBER DE-FG05-88ER40461

DASTRIBUTHOA OF THS DOCUMENT IS UNLMITED 


\section{DISCLAIMER}

This report was prepared as an account of work sponsored by an agency of the United States Government. Neither the United States Government nor any agency thereof, nor any of their employees, makes any warranty, express or implied, or assumes any legal liability or responsibility for the accuracy, completeness, or usefulness of any information, apparatus, product, or process disciosed, or represents that its use would not infringe privately owned rights. Reference berein to any specific commercial product, process, or service by trade name, trademark, inanufacturer, or otherwise does not necessarily constitute or imply its endorsement, recommendation, or favoring by the United States Government or any agency thereof. The views and opinions of authors expressed herein do not necessarily state or reflect those of the United States Government or any agency thereof. 


\section{DISCLAIMER}

Portions of this document may be illegible in electronic image products. Images are produced from the best available original document. 


\section{INTRODUCTION}

This report details research progress and results obtained during the ten period from June 1, 1988 through May 31, 1998. The research project, entitled "Theoretical Nuclear Reaction and Structure Studies Using Hyperons and Photons", was supported by grant DE-FG05-88ER40461 between North Carolina State University (NCSU) and the United Stateš Department of Energy (DOE). In compliance with grant requirements the Principal Investigator, Professor Stephen R. Cotanch, has conducted a research program addressing theoretical investigations of reactions involving hyperons and photons. The Principal Investigator has devoted to this program 50\% of his time during the academic year and $100 \%$ of his time in the summer. Highlights of significant research results are briefly summarized in the following three sections which respectively correspond to the three sub-programs of this project.

\section{ELEMENTARY HADRON STRUCTURE}

This project seeks to further the understanding of hadron structure within the framework of Quantum Chromodynamics (QCD) and QCD based models and gain dynamic insight into the color confinement mechanism. The key accomplishments are briefly summarized:

1) a comprehensive low mass meson and baryon analysis of static and dynamic properties using alternative relativistic quark models has been performed;

2) a long standing anomaly has been resolved by obtaining a simultaneous description of the neutron charge radius and the neutron to proton structure function ratio for Bjorken variable $\mathrm{x}$ near 1;

3) the correct criterion to validate the perturbative QCD contribution for exclusive processes has been determined;

4) QCD sum rules have been used to calculate the light quark energy distribution in a heavy meson leading to significant differences from that predicted by a simple quark/antiquark structure;

5) a variable front quantization constituent quark model was developed to determine quantization dependence. This permitted rotating the null plane continuously from the lightcone framework to the instantaneous formulation. Calculated observables showed little sensitivity provided relativity was properly implemented. Most significantly, it was ascertained that relativity was essential for any hadronic form factor calculation. We 
concluded that, within a relativistic quantum mechanical framework (not field theory), the two formulations are phenomenologically equivalent;

6) the Bakamjian-Thomas construction was utilized to study nucleon structure in the relativistic quark model. Electromagnetic matrix elements were calculated using one-body current operators and the Lorentz symmetry breaking resulting from such an approximation was investigated in detail for both the light front and instant quantization limits;

7) leading order QCD corrections have been calculated for transition form factors between heavy and light mesons. The corrections are large and dependent upon the meson wave functions. Results are in reasonable agreement with recent branching ratio measurements for $\mathrm{B}->\pi \mathrm{l} v$ reported by Cleo;

8) a new, comprehensive relativistic many-body approach to hadron structure based upon the Coulomb gauge QCD Hamiltonian has been developed. Initial application has been made to compute the glueball spectra and remarkable agreement has been obtained with lattice theory;

9) a new renormalization procedure which generates an effective Hamiltonian for both the gluon and quark sector has been developed. The formulation is in the Coulomb gauge where the QCD Hamiltonian is renormalizable and the Gribov problem can be resolved. We utilize elements of the Glazek and Wilson regularization method but now introduce a continuous cut-off procedure which eliminates non-local counterterms. The effective Hamiltonian is then derived to second order in the strong coupling constant. The resulting renormalized Hamiltonian provides a realistic starting point for approximate many-body calculations of hadronic properties for systems with both quark (mesons and baryons) and explicit gluon degrees of freedom (glueballs and hybrids).

Because of our interest and excitement with the relativistic many-body hadron approach (above items 8 and 9), it is worthwhile to comment further on this project. The effective field theory Hamiltonian is chirally symmetric but using the BCS variational approach chiral symmetry breaking naturally occurs and both the quarks and gluons acquire constituent masses, roughly 100 $\mathrm{MeV}$ and $900 \mathrm{MeV}$, respectively. Further, both quark and gluon condensates are calculated yielding $-(100 \mathrm{MeV})^{3}$ and $.012 \mathrm{GeV}^{4}$, respectively. The glueball spectra was then computed using the traditional nuclear TDA and RPA approaches. Finally, it is noteworthy that we find only that the RPA preserves the Nambu-Goldstone realization of chiral symmetry. In particular the Gell-Mann-Oaks-Renner relation emerges which yields zero mass "chiral" pions in the limit the current quark mass goes to zero.

For further details consult the publications listed at the end of this report. 


\section{ELECTROMAGNETIC PRODUCTION OF STRANGENESS}

This project addresses systems involving the strange quark (hyperons), as well as hidden strangeness, and investigates strange and non-strange meson electromagnetic production and radiative capture processes. The motivation and philosophy is to study the novel physics associated with the s quark in the nucleon (hidden strangeness), hyperon (explicit strangeness), few hyperon systems and hypernuclei and to also exploit the unique, attractive features of these systems to search for exotic resonances and further understand fundamental dynamics, reaction mechanisms, and many-body structure. In the project period we have obtained and published several interesting findings:

1) developed a unified model, based on Quantum Hadrodynamics (QHD), which yielded a comprehensive theoretical description of the world's data for elementary kaon electromagnetic production and radiative capture forming $\Lambda, \Lambda(1405)$ and $\Sigma$ baryons; 2) performed an analysis of the first elementary kaon electroproduction measurements at Jefferson lab involving $\mathrm{L} / \mathrm{T}$ separation into longitudinal and transverse cross sections; 2) conducted an analysis of the first kaon electroproduction measurements at Jefferson lab from a deuteron target, $\mathrm{d}\left(\mathrm{e}, \mathrm{e}^{\prime} \mathrm{K}^{+}\right) \mathrm{YN}$ for $\mathrm{YN}=\Lambda \mathrm{n}, \Sigma^{0} \mathrm{n}$ and $\Sigma^{-} \mathrm{p}$ and investigated final state baryon interactions;

3) completed a $\phi$ meson photoproduction analysis for the reaction $\mathrm{p}(\gamma, \phi) \mathrm{p}$ combined with new predictions for $\mathrm{N}\left(\pi, \mathrm{e}^{+} \mathrm{e}^{-}\right) \mathrm{N}$;

4) used vector meson dominance to investigate the strangeness content of the nucleon;

5) initiated theoretical work in computing the $\mathrm{g}_{\phi \mathrm{NN}}$ coupling constant;

6) obtained preliminary exact few-body structure calculations for ${ }^{4} \mathrm{He}\left(\mathrm{e}, \mathrm{e}^{\prime} \mathrm{K}^{+}\right)^{4} \mathrm{H}_{\Lambda}$.

Our QHD model includes the constraints due to crossing symmetry and duality and was also used for $\mathrm{p}(\gamma, \phi) \mathrm{p}$ and $\mathrm{N}\left(\pi, \mathrm{e}^{+} \mathrm{e}^{-}\right) \mathrm{N}$. The strangeness content of the nucleon study centered upon documenting the utility of the $\mathrm{N}\left(\pi, \mathrm{e}^{+} \mathrm{e}^{-}\right) \mathrm{N}$ reaction. We demonstrated through $\mathrm{QHD}$ calculations and extended vector meson dominance that measurements of this process will provide new information about the nucleon electromagnetic form factors in the time-like region which is kinematically inaccessible using two-body final state reactions. Most significantly we predict dramatic, three-order of magnitude narrow resonances from vector meson $(\rho, \omega$ and $\phi)$ contributions. Including $\phi N$ coupling in the form factor yields a unique dual peak experimental cross section signature for assessing possible OZI suppression or evasion and the related 
strangeness content of the nucleon. This work is in conjunction with experimentalists at GSI and BNL who are planning to perform such measurements.

Our deuteron target calculation, $\mathrm{d}\left(\mathrm{e}, \mathrm{e}^{\prime} \mathrm{K}^{+}\right) \mathrm{YN}$, entails a coupled channels final state interaction study using potential D of Nagels and de Swart for the three channels $\Lambda \mathrm{n}, \Sigma^{0} \mathrm{n}$ and $\Sigma^{-} \mathrm{p}$. We also performed extensive, large-scale precision calculations which indicated that exclusive and not inclusive measurements will be needed to extract final state information relating to $\mathrm{YN}$ interactions. Our work is being coordinated with experimentalists at CEBAF in support of additional approved experiments.

\section{MEDIUM ENERGY PHOTONUCLEAR REACTIONS}

This is a long term, continuing program addressing many-body nuclear structure and baryon electromagnetic currents in the nucleus. We performed large-scale continuum shell model calculations for $(\gamma, \mathrm{N})$ and $(\mathrm{N}, \gamma), \mathrm{N}=\mathrm{p}$ and $\mathrm{n}$, reactions at low and medium energies spanning the $\Delta$ isobar region. This is a microscopic formulation in which a realistic many-body Hamiltonian $\mathrm{H}$ for the $\mathrm{A}$ baryon system is diagonalized in a large Banach space (not Hilbert since the continuum basis states are not square integrable) spanned by products of continuum single-particle states and known bound many-body eigenfunctions of the A-1 system. The Hamiltonian contains a realistic effective interaction with finite range, spin, isospin and tensor components. The code is flexible and can accommodate a variety of two-body forces including those based on multi-boson exchange. Diagonalizing $\mathrm{H}$ in this space generates a large set of coupled inhomogeneous integrodifferential equations in two variables ( $r$ and $r$ due to non-localities) to be solved, subject to appropriate scattering boundary conditions, for the unknown partial-wave scattering functions. All non-localities are retained in the radial kernels due to wavefunction antisymmetrization, Pauli blocking and exchange. Since the large number (up to 100 currently) of scattering channels rigorously account for loss of flux from the elastic channel there is no direct need for phenomenological absorption and $\mathrm{H}$ therefore remains Hermitian which assures proper wavefunction orthogonality that is especially important in photonuclear reactions. Further, the code rigorously respects unitary and gauge-invariance.

Initially we determined the parameters in our effective interaction by obtaining a description of the giant resonance for both Carbon and Oxygen. We then extended our Carbon, ${ }^{11} \mathrm{~B}(\mathrm{p}, \gamma)^{12} \mathrm{C}$, ${ }^{11} \mathrm{~B}(\mathrm{p}, \gamma){ }^{12} \mathrm{C}^{*}\left(2^{+}\right)$, and Oxygen, ${ }^{16} \mathrm{O}(\gamma, \mathrm{p})^{15} \mathrm{~N},{ }^{16} \mathrm{O}(\gamma, \mathrm{n})^{15} \mathrm{O}$, calculations to higher energies and included additional multipoles. For medium energy photonuclear reactions it is crucial to include 
all electromagnetic multipole transitions up to $\mathrm{E} 10$ and $\mathrm{M} 9$ for the ${ }^{12} \mathrm{C}$ calculation (for proton energy $=178 \mathrm{MeV}$ ) and E17, M15 for the higher energy ${ }^{16} \mathrm{O}$ (for photon energy up to $400 \mathrm{MeV}$ ) analysis. This assures full numerical convergences of the multipole expansion for the electromagnetic field. Note that for each $\mathrm{J} \pi$ multipole we solve $\mathrm{N}$ coupled, non-local equations (which for accuracy must be solved $\mathrm{N}$ times to obtain the proper linear combination) with a radial mesh size that must become finer as both the number of channels and energies increase. For ${ }^{16} \mathrm{O}$ we obtained a good description of the forward Bates data using $A=151 \mathrm{p}-1 \mathrm{~h}$ basis states (only the $1 / 2^{-}$ground state at first excited $3 / 2^{-15} \mathrm{O}$ and ${ }^{15} \mathrm{~N}$ state were included). This calculation requires (with no $\Delta$ isobars) 12 coupled equations for the $32 \mathrm{~J} \pi$ multipoles included an( $\mathrm{d}$ a radial mesh of .04 fermi. For ${ }^{12} \mathrm{C}$, which is not a closed shell nucleus, especially the $2^{+}$excited state, we needed to use an extended multiparticle - multihole basis for the $\mathrm{A}=11$ system and included the first 5 excited states in ${ }^{11} \mathrm{C}$ and ${ }^{11} \mathrm{~B}\left(1 / 2_{1}^{-}, 3 / 2_{2}^{-}, 5 / 2_{1}^{-}, 3 / 2_{2}^{-}, 7 / 2_{1}^{-}\right)$. The extended model calculation requires up to 48 channels for each $\mathrm{J}^{\pi}$ (again with a small radial mesh). Hence the calculation is tantamount to inverting a very large supermatrix of the order 20,000 by 20,000 for each $J \pi$ at each energy. These calculations were too taxing for our theory group computer and were predominantly performed on the N. C. and Livermore CRAYs . The significant result of our conventional calculation without exchange currents is, for the first time, a simultaneous description of both $(\gamma, \mathrm{p})$ and $(\gamma, \mathrm{n})$, including cross section magnitude, with one unified model. The effects from $\Delta$ isobars and pion (pole term and the Kroll-Ruderman) exchange currents were in general small except at large energies and momentum transfers. Polarization observables were much more sensitive to exchange currents than cross sections.

For the medium energy ${ }^{11} \mathrm{~B}(\mathrm{p}, \gamma)^{12} \mathrm{C}$ from the Svedberg Laboratory in Uppsala, Sweden we also obtained a good description for transitions to the ground and first three excited states for proton energies of 98 and $176 \mathrm{MeV}$. Although we again included $\Delta$ isobars, the pion pole term and the Kroll-Ruderman exchange currents, we find the predominant mechanism involves the photon coupling to the one-body nucleon current. Exchange current effects are more significant for higher momentum transfers, spin and polarization observables and perhaps two-nucleon photoknockout.

This project has supported experimental investigations at the Bates Laboratory, the Indiana University Cyclotron, the Svedberg Laboratory in Uppsala, Sweden, future CEBAF measurements, and TUNL collaborative experiments at LEGS. 


\section{SERVICE AND NON-RESEARCH ACTIVITIES}

In addition to research, the $\mathrm{Ph}$. $\mathrm{D}$. investigators supported by the grant contributed to the nuclear community in service. In particular, they organized conferences, workshops and summer schools, refereed numerous papers and proposals, and participated in the Institute for Nuclear Theory (INT) programs. Finally, the principle investigator co-founded the weekly Triangle Nuclear Theory (TNT) seminar series, which is supported by DOE, and continues to be very successful. The stimulating talks by renown theorists not only provided an informative forum for educating triangle nuclear physicists but also helped foster interactions between the theorists at each triangle university. The speakers were:

J. Piekarewicz (SCRI, Florida State), C. Carraro (Harvard), J. Engel (Bartol Research Institute), T. Biro (Univ. Giessen, Germany), S. Pollock (NIKHEF), D. Leinweber (Maryland), A. Szczepaniak (NCSU), E. Swanson (MIT), M. Chu (Caltech), D. Richards (Univ. Edinburgh, Scotland), C. Morningstar (SLAC), P. Jain (Univ. Kansas), S. Schramm (Indiana Univ.), A. Harindranath (Ohio State), D. Min (Seoul National Univ.), I. Kogan (Princeton), W. Haxton (Univ. Washington), T. Cohen (Maryland), D. Hartman (Clemson), P. Sikivie (Univ. Florida), B. Grinstein (SSC), M. Musolf (Old Dominion/CEBAF), J. Clark (Washington Univ.), F. Tabakin (Univ. Pittsburgh), A. Sandorfi (Brookhaven), S. Wallace (Maryland), R. Malaney (Univ. Toronto). Bruce Barrett (Arizona), Ubirajara van Kolck ( Univ. of Washington), Stuart Pittel (Bartol Res. Institute), Henry Weller (Duke Univ.), Don Lehman (George Washington Univ.), Jim Napolitano ( RPI), Vladimir Braun (MPI Heidelberg), Jitendra Parikh (Ahmedabad), Robert Perry (Ohio State), Alan White (Argonne), Fritz Coester (Argonne), David Kosower (Saclay), Gerry Brown (Stony Brook), Ian Towner (Chalk River), Brian Serot (Univ. of Indiana), Harry Lee (ANL), Mahir Hussein (Sao Paulo and MIT), Barry Holstein (Univ. of Massachusetts), Rudy Hwa (Univ. of Oregon), Ted Barnes (ORNL), Lenny Kisslinger (Carnegie Mellon Univ.), George Bertsch (Univ. of Washington). David London (Montreal), Keh-Fei Liu (Kentucky), Eric Braaten (Northwestern), Frank Iachello (Yale), Brad Keister (NSF), Mikkel Johnson (Los Alamos), Michael Strayer (Oak Ridge), Miklos Gyulassy (Columbia), Witold Nazarewicz (Oak Ridge), Russel Betts (Argonne), 
Rocco Schiavilla (CEBAF), Da Hsuan Feng (Drexel), Ernest Henley (Univ. Washington), Ulf Meissner (Univ. Bonn), Mikhail Shmatikov (Kurchatov Instit., Moscow), Alex Krasnitz (Niels-Bohr Instit.), Robert Singleton (Boston Univ.), Joseph Ginocchio (Los Alamos), Craig Roberts (Argonne), Larry McLerran (Minnesota), Michael Guidry (Tennessee), Charles Horowitz '(Indiana), Peter Herczeg (Los Alamos), James Vary (Iowa State), Warre Buck (CEBAF and Hampton Univ.). Scott Pratt (Michigan State), Paulo Bedaque (MIT), Stan Brodsky (SLAC), Steve Tomsovic (Washington State), David Kaplan (INT, Seattle), Petr Vogel (Caltech), Nimai Mukhopadhyay (RPI), Daniel Boyanovsky (Univ. Pittsburgh), Richard Furnnstahl (Ohio State), Torleif Ericson (CERN), Jochen Rau (ECT,* Trento), Nora Brambilla (Univ. Milano), Edward Shuryak (Stony Brook), Jose Goity (TJNAF), John Harris (Yale), David Dean (Oak Ridge), Cornelius Benhold (George Washington), John Hiller (Univ. Minnesota-Duluth) and Sherman Frankel (Univ. Pennsylvania). Yuri Kovchegov (Columbia Univ.), Dan Riska (Helsinki), Eric Laenen (SUNY), Fred Cooper (LANL), Raju Venugopalan (Niels Bohr Institute), Brad Keister (Carnegie Mellon) Mark Alford (IAS, Princeton), Shailesh Chandrasekharan (MIT), Philip Page (Jefferson Lab), Jac Verbaarschot (SUNY), Arthur Kerman (MIT), Franz Gross (William\&Mary), Alan Chodos (Yale), Fred Myhrer (South Carolina), David Robertson (Ohio State), Chuang Chan (Univ. of Washington). Hee Kwan Han (Korea), Veljko Dmitrasinovic (USC), Andrei Afanasev (NCCU), Brad Meyer (Clemson), Michael Mattis (LANL), Wolfgang Poeschl (Duke), Susan Gardner (Kentucky), Tom Cohen (Maryland), Ken Barish (UCLA), Jacek Dobaczewski (ORNL/Warsaw), Maurice Goldhaber (Brookhaven), Daniel Zwanziger (NYU), Claudio Coriano (TJNAF), Dirk Hueber (Los Alamos), Dinesh Srivastava (Duke), Randy Lewis (Regina/TJNAF), Mike Luke (Toronto), Frank Woelz (Iowa State University), Mikkel Johnson (Los Alamos/ CMU), Mike Pichowski (Florida State), Joe Ginocchio (Los Alamos), Andrei Leonidov (Lebedev Institute), Emilio Ribeiro (Lisbon), Wick Haxton (INT/Univ. of Washington), Carl Shakin (Brooklyn), Don Robson (Florida State), Nathan Isgur (CEBAF).

In addition many of these speakers spent multi-day, productive visits with the NCSU group. 


\section{PAPERS PUBLISHED IN THE REPORT PERIOD}

(only by the Principal Investigator)

"Crossing Constraints for Hyperon Reactions", C.-R. Ji and S. R. Cotảnch, Phys. Rev. C38, 2691 (1988).

"Electromagnetic Production of Mesons", S. R. Cotanch and S. S. Hsiao, Proceedings of the Third Workshop on Perspectives in Nuclear Physics at Intermediate Energies, International Center for Theoretical Physics, Trieste, Italy. eds. S. Boffi, C. Ciofi degli Atti and M. M. Giannini, World Scientific, 141 (1988).

"Strangeness Production Studies at CEBAF", S. R. Cotanch, Proceedings of the International Conference on Medium- and High-Energy Nuclear Physics, eds. W-Y. Pauchy Hwang, Keh-Fei Liu and Y. Tzeng, World Scientific (Singapore), 666 (1989).

"Deuteron Electrodisintegration Through Kaon Production", S. S. Hsiao and S. R. Cotanch, Proceedings of the International Conference on Medium- and High-Energy Nuclear Physics, eds. W-Y. Pauchy Hwang, Keh-Fei Liu and Y. Tzeng, World Scientific (Singapore), 368 (1989).

"A Crossing-Consistent Analysis of Kaon Photoproduction and Radiative Capture", R. A. Williams, C.-R. Ji and S. R. Cotanch, Proceedings of the 12th International Conference on Few Body Problems in Physics, ed. B. K. Jennings, Triumph, Vancouver, I 25 (1989).

"Crossing-Consistent Analysis of Kaon Photoproduction and Radiative Capture", R. A. Williams, C.-R. Ji and S. R. Cotanch, Phys. Rev. D41, 1449 (1990).

"Simple Relativistic Quark Model Analysis of Flavored Pseudoscalar Mesons", C.-R. Ji and S. R. Cotanch, Phys. Rev. D41, 2319 (1990).

"The Importance of Polarization in Kaon Photoproduction and Radiative Capture", S. R. Cotanch, R. A. Williams and C.-R. Ji, Colloque de Physique C6, 491 (1990).

"Understanding Electroweak Couplings of the Pion as a qq̄ Composite", C.-R. Ji and S. R. Cotanch, Phys. Rev. Lett. 64, 1848 (1990).

"Crossing and Duality Study of $\Lambda, \Sigma^{\circ}$, and $\Lambda$ (1405) Production by Kaon Photoproduction and Radiative Capture", R. A. Williams, C.-R Ji and S.R. Cotanch, Phys. Rev. C43, 452 (1991).

"Radiative Proton Capture to Individual States in ${ }^{12} \mathrm{C}$ ", B. Höistad, E. Nilsson, J. Thun, S. Dahlgren, S. Isaksson, G.S. Adams, C. Landberg, T. Bright and S.R. Cotanch, Phys. Lett. B276, 294 (1992).

"Light-Cone Quark Model Axial Vector Meson Wavefunction", C.-R. Ji, P. L. Chung and S.R. Cotanch, Phys. Rev. D45, 4214 (1992). 
"Hyperon Electroproduction in a Crossing and Duality constrained Model", R.A. Williams, C.-R. Ji and S.R. Cotanch, Phys. Rev. C46, 1617 (1992).

"Radiative Proton Capture and Exchange Currents in Light Nuclei", B. Höistad, S. Isaksson, E. Nilsson, J. Thun, G.S. Adams, C. Landberg, T. Bright and S.R. Cotanch, Nucl. Phys. A553, 543(1993).

"Kaon Electromagnetic Production and Radiative Capture Near Hyperon Thresholds", S.R. Cotanch, R.A. Williams and C.-R. Ji, Physica Scripta 48, 217(1993).

"Simultaneous Microscopic Description of ${ }^{16} \mathrm{O}(\gamma, \mathrm{N})$ Including $\Delta$ Degrees of Freedom", T.B. Bright and S.R. Cotanch, Phys. Rev. Lett. 71, 2563(1993).

"Kinematically Accessible Vector Meson Resonance Enhancements in $\mathrm{p}\left(\mathrm{K}^{-}, \mathrm{e}^{+} \mathrm{e}^{-}\right) \Lambda, \Sigma, \Lambda(1405)$ ", R. A. Williams, C.-R. Ji and S.R. Cotanch, Phys. Rev.C48, 1318 (1993).

"Radiative Proton Capture in Light Nuclei at Intermediate Energies", B. Höistad, R. Johansson, E. Nilsson, J. Thun, G.S. Adams, T. Bright and S.R. Cotanch, Proceedings of the 8th International Symposium on Capture Gamma Ray Spectroscopy, Fribourg (World Scientific), 530(1994).

"Generalized Relativistic Meson Wave Function", A. Szczepaniak, C.-R. Ji and S. R. Cotanch, Phys. Rev. D49, 3466(1994).

"Crossing Constraint in Kaon Production and Capture", C.-R. Ji, R. A. Williams and S. R. Cotanch, Proceedings of the 6th Symposium on Nuclear Physics, Muju, Korea, eds. D.-P. Min and B.-Y. Park (Han Lim Won), 156 (1994).

"Significant Nonvalence Components in Heavy Quark Symmetry", A. Szczepaniak, C.-R. Ji and S. R. Cotanch, Phys. Rev. Lett. 72, 2538(1994).

"An Overview of Electromagnetic Hadronic Physics Involving Strangeness", S. R. Cotanch, Revista Mexicana de Fisica 40, 297(1994).

"On the Constituent Quark Structure", A. Szczepaniak, C.-R. Ji and S. R. Cotanch, AIP Conference Proceedings 334, 647(1995).

"Relativistic Quark Model Description of Baryon Properties", S. R. Cotanch, A. Szczepaniak and C.-R. Ji, AIP Conference Proceedings 334, 575(1995).

"Quantization Dependence in a Constituent Quark Model", A. Szczepaniak, C.-R. Ji and S. R. Cotanch, Phys. Rev. D52, 5284(1995).

"Relativistic Quark Model Baryon Analysis", S. R. Cotanch, A. Szczepaniak and C.-R. Ji, FewBody Systems Suppl.(Springer-Verlag) 8, 354(1995).

"Nucleon Structure in a Relativistic Quark Model," A. Szczepaniak, C.-R. Ji and S. R. Cotanch, Phys. Rev. C52, 2738(1995).

"Glueball Spectroscopy in a Relativistic Many-Body Approach to Hadron Structure", A. Szczepaniak, E. Swanson, C.-R. Ji and S. R. Cotanch, Phys. Rev. Lett. 76, 2011(1996). 
"Radiative Proton Capture to Discrete States in ${ }^{12} \mathrm{C}$ at 98 and $176 \mathrm{MeV}$ ", T. B. Bright, B. Hoistad, R. Johansson, E. Traneus and S. R. Cotanch, Nucl. Phys. A603, 1 (1996).

"Nucleon Strangeness Content through Vector Meson Dominance", R. A. Williams and S. R. Cotanch, Phys. Rev. Lett. 77, 1008(1996).

"Light-Cone Quark Model of Mesons", C.-R. Ji, A. Szczepaniak and S. R. Cotanch, Particle Theory and Phenomenlogy, eds. K. E. Lassila, J. Qiu, A. Sommerer, G. Valencia, K. Whisnant and B. -L. Young (World Scientific), 311 (1997).

"Many-Body Hamiltonian Approach to QCD", S. R. Cotanch, A. Szczepaniak, E. Swanson and C.-R. Ji, Quark Confinement and the Hadron Spectrum II, eds. N. Brambilla and G. M. Prosperi, World Scientific, 342 (1997).

"Radiative Proton Capture to Discrete States in ${ }^{13} \mathrm{~N}$ at 98 and $176 \mathrm{MeV}$, T. B. Bright, B.Hoistad, R. Johansson, E. Traneus and S. R. Cotanch, Nucl. Phys. A621, 754 (1997).

"Many-Body Hamiltonian Approach to QCD: Applications", A. Szczepaniak, E. Swanson, C.-R. Ji and S. R. Cotanch, AIP Conference Proceedings 412, 733 (1997).

"Nucleon Strangeness Content through Vector Meson Dominance", S. R. Cotanch and R. A. Williams, Particles and Nuclei PANIC '96, eds. C. E. Carlson and J. J. Domingo, 251 (1997).

"Glueball Spectroscopy in a Many-Body QCD Hamiltonian Approach", S. R. Cotanch, A. Szczepaniak, E. Swanson and C.-R. Ji, AIP Conference Proceedings 412, 515 (1997).

"Probing Nucleon Strangeness using Vector Meson Dominance", S. R. Cotanch and R. A. Williams, Nucl. Phys. A631, 478 (1998).

"QCD Hamiltonian Approach for the Glueball Spectrum", S. R. Cotanch, A. Szczepaniak, E. Swanson and C.-R. Ji, Nucl. Phys. A631, 640 (1998).

"Kaons and Glueballs at Jefferson Lab: a Strange, Sticky but Colorful Production", S. R. Cotanch, Proceedings of the VI Hadron Physics Conference (World Scientific), in press (1998).

\section{BOOK CHAPTERS}

"Hypernuclear Physics and Kaon Electromagnetic Production", S. R. Cotanch, in "Quarks, Mesons and Nuclei" Vol. II, eds. W-Y. P. Hwang and E. M. Henley, World Scientific (Singapore), 190 (1989).

"Large Scale Continuum Shell Model Calculations for Photonuclear Reactions with $\Delta$ Isobars and Exchange Currents", S. R. Cotanch and T. B. Bright, book chapter in Contemporary Nuclear Shell Models, eds. X. W. Pan, D. H. Feng and M. Vallieres, Lecture Notes in Physics, Springer-Verlag, 172 (1997). 


\section{INVITED LECTURES}

"Hypernuclear Physics and Kaon Electromagnetic Production", Spring School on Medium and High-Energy Nuclear Physics, Taipei, Taiwan (1988).

"Theoretical Nuclear and Particle Physics" Korean High School Scî́ence Teachers Program. The lecture was filmed, with translations, at the Science House, North Carolina State University ( August, 1995).

\section{INVITED PAPERS}

(only by the Principal Investigator)

"Strangeness Production Studies at CEBAF", International Conference on Medium and HighEnergy Nuclear Physics, Taipei, Taiwan (1988).

"Electromagnetic Production of Hyperons and Hypernuclei", CEBAF Workshop on End Station Design, Newport News, Va, 1989.

"Strangeness Electromagnetic Production", Long Range Plan Workshop on Very Light Nuclei, Duke University, Durham, N.C., 1989.

"The Importance of Polarization in Kaon Photoproduction and Radiative Capture", Seventh International Conference on Polarization Phenomena in Nuclear Physics, Paris, France (1990).

"Hyperon Electromagnetic Production Studies at CEBAF", Ninth Nordic Meeting on Intermediate and High Energy Nuclear Physics, Gräftavallen, Sweden (1991).

"Continuum Shell Model Approach to Medium Energy Photonuclear Reactions", Ninth Nordic Meeting on Intermediate and High Energy Nuclear Physics, Gräftavallen, Sweden (1991).

"Strangeness Electromagnetic Production at CEBAF", International Workshop on Meson Production, Interaction and Decay, Cracow, Poland (1991).

"Simple Light-Cone Quark Model Description of Meson Properties", 13th International Conference on Few Body Problems in Physics, Adelaide Australia (1992).

"Continuum Shell Model Approach to Medium Energy Photonuclear Reactions", Symposium on Nuclear Reactions to Commemorate the 65th Birthday of G.R. Satchler, Oak Ridge, TN(1992). 
"Kaon Electromagnetic Production and Radiative Capture Near Hyperon Thresholds", Second International Conference on Particle Production Near Threshold, Uppsala Sweden (1992).

"Relativistic Quark Model Analysis of Flavored Mesons", Tenth Nordic Meeting on Intermediate and High Energy Nuclear Physics, Gräftavallen, Sweden (1993).

"The Strangeness and Hidden Strangeness Program at CEBAF," Overview moderator talk at the CEBAF User's Retreat, Snowshoe, WVA (1993).

"Elementary Kaon Electromagnetic Production and Radiative Capturể", Nato Advance Study Institute on Hot and Dense Nuclear Matter, Bodrum, Turkey (1993).

"High Momentum Studies of Strangeness at CEBAF", CEBAF Fall Strangeness Workshop, Newport News, VA (1993).

"An Overview of Electromagnetic Hadronic Physics Involving Strangeness", Seventeenth Symposium on Nuclear Physics at Oaxtepec, Morelos, Mexico (1994).

"Relativistic Quark Model Description of Baryon Properties", 14th International Few Body Conference, Williamsburg, VA (1994).

"Relativistic Quark Model Baryon Analysis", 15th European Conference on Few-Body Problems in Physics, Peniscola, Spain (1995).

"Baryons in Relativistic Quark Model", International Nuclear Physics Conference, Beijing, China (1995).

"Proton Strangeness Studies using the $\mathrm{p}\left(\pi^{-}, \mathrm{e}^{+} \mathrm{e}^{-}\right)$and $\mathrm{p}\left(\mathrm{K}^{-}, \mathrm{e}^{+} \mathrm{e}^{-}\right)$reactions", Ninth Journees des Theoriciens Saturn, Saclay, France (1995).

"Kaon Electromagnetic Production and Radiative Capture", CEBAF/INT Workshop on Open Issues in Hypernuclear Physics, CEBAF, Newport News, VA (1995).

"Large-Scale Continuum Shell Model Calculations for Photonuclear Reactions with $\Delta$ Isobars and Exchange Currents", Workshop on Contemporary Nuclear Shell Model, Philadelphia, PA (1996).

"Nucleon Strangeness Content through Vector Meson Dominance", S. R. Cotanch, Workshop on AGS Experiments for the 21st Century (AGS-2000), Brookhaven National Laboratory, Upton, N. Y. (1996).

"Nucleon Strangeness Content through Vector Meson Dominance", S. R. Cotanch, XIV International Conference on Particles and Nuclei (PANIC), Williamsburg, VA (1996).

"Many-Body Hamiltonian Approach to QCD", S. R. Cotanch, International Workshop on Quark Confinement and the Hadron Spectrum II, Como, Italy, (1996).

"Probing Nucleon Strangeness using Vector Meson Dominance", S. R. Cotanch, 15th International Conference on Few-Body Problems in Physics, Groningen, The Netherlands (1997).

"Glueball Spectroscopy in a Many-Body QCD Hamiltonian Approach", 6th Conference on the Intersections of Particles and Nuclei, S. R. Cotanch, Big Sky, Montana (1997). 
"Large-Scale Continuum Shell Model Calculations for Photonuclear Reactions with $\Delta$ Isobars and Exchange Currents", S. R. Cotanch, Program on Nuclear Structure Studies with Electrons (INT97-2), Institute for Nuclear Theory, Seattle, Washington (1997).

"Nucleon Strangeness Content through Vector Meson Dominance, S. R. Cotanch, 6th Conference on the Intersections of Particles and Nuclei, Big Sky, Montana (1997).

"Kaon Electromagnetic Production and Glueballs, a strange, sticky, but colorful way to do physics", S. R. Cotanch, Theoretical Symposium to Commemorate the 60th Birthday of Donald Robson, Athens, Georgia (1997).

"QCD Hamiltonian Approach for the Glueball Spectrum", S. R. Cotanch, 15th International Conference on Few-Body Problems in Physics, Groningen, The Netherlands (1997).

"Kaons and Glueballs at Jefferson Lab: a Strange, Sticky but Colorful Way to do Physics", S. R. Cotanch, VI Hadron Physics 1998, Florianopolis, Brazil (1998).

Also, there were several abstracts detailing contributed papers at meetings. Further, the investigators presented many colloquiums and seminars (nationally and internationally). These references are available upon request. 